\title{
INFLUÊNCIA DA INTERNACIONALIZAÇÃO E DA INOVAÇÃO NA COMPETITIVIDADE EMPRESARIAL
}

\author{
Antonio Rodrigues Albuquerque Filho, Maria Macileya Azevedo Freire, Marcia Martins Mendes De Luca, \\ Alessandra Carvalho de Vasconcelos ${ }^{1}$ \\ Universidade Federal do Ceará - UFCE, Fortaleza, Ceará, (Brasil)
}

\section{DETALHES DO ARTIGO}

Recebido: 31 de janeiro de 2019

Aceito: 25 de setembro de 2019

Disponível online: 01 de jan. de 2020

Sistema de revisão "Double blind review"

Editor Científico

Ilan Avrichir

\section{Palavras-chaves:}

Internacionalização

Inovação

Competitividade

BRICS

\section{RESUMO}

Objetivo: Investigar a influência da internacionalização e da inovação na competitividade em empresas de países do BRICS listadas na Nyse, sob a perspectiva da VBR.

Método: De cunho descritivo e abordagem quantitativa, a pesquisa utiliza o teste de diferenças entre médias, a correlação e a regressão linear múltipla com dados em painel, para análise de dados de 2015 a 2017, de 64 empresas não financeiras sediadas em países do BRICS listadas na Nyse.

Principais resultados: Corroborando os achados constatados nos testes de diferenças entre médias e correlação de Pearson, os resultados da regressão indicam que os indicadores de internacionalização e os de inovação não exercem influência sobre a competitividade das empresas. Ressalte-se, porém, que, na presença de estratégias de inovação, a rentabilidade se mostra relevante, denotando que quanto maior for a rentabilidade da organização, menor será o seu poder de competitividade.

Relevância/originalidade: Apesar de individualmente a internacionalização e a inovação serem temas amplamente discutidos na literatura, é relevante que sejam explorados na perspectiva da competitividade empresarial, uma vez que são essenciais para o fortalecimento da economia e apontados como de grande valor organizacional. Outrossim, poucas pesquisas analisaram empiricamente a competitividade tendo como proxy o índice de Herfindahl-Hirschman.

Contribuições teóricas/metodológicas: Destaca-se a contribuição do estudo para o campo acadêmico ao ponderar a internacionalização e a inovação como métricas multidimensionais para análise do envolvimento internacional das empresas e da adaptação estratégica de recursos como fontes de vantagem competitiva.

\section{INTRODUÇÃO}

Nas últimas décadas, a economia mundial vem experimentando significativas mudanças. Em um mercado cada dia mais competitivo e globalizado, torna-se essencial que as empresas procurem adotar estratégias que lhes proporcionem maior desenvolvimento (Browm \& Kimbrough, 2011), possibilitando-lhes destacar-se quanto à capacidade e eficiência para criar, expandir e aplicar recursos (Perez \& Famá, 2015), requerendo, dessa forma, padrões de conduta capazes de assegurar diferenciais de competitividade e sobrevivência (Perez \& Famá, 2015).

Ressalte-se também que as companhias destacam-se competitivamente quando adquirem resultados satisfatórios a partir da adoção de práticas aceitas e valorizadas no ambiente em que atuam, motivo pelo qual muitas procuram se referenciar nos exemplos de sucesso (Moura, Mecking \& Scarpin, 2013). Assim, as estratégias competitivas refletem a maneira como as empresas distinguem-se de suas concorrentes, utilizando seus próprios mecanismos para obter maior eficiência em termos de liderança de custos (Vargas, 2015) e/ou maior produtividade por meio da diferenciação de produtos e serviços ofertados (Block, Kohn, Miller \& Ullrich, 2015).

Dentre as diversas estratégias competitivas, destacam-se a internacionalização e a inovação, apontadas como vetores de vantagem competitiva e que agregam valor ao negócio. A internacionalização é motivada pela necessidade de recursos, mercados e ativos estratégicos (Stal, 2010), enquanto a inovação, considerada um recurso indispensável em mercados altamente competitivos, é impulsionada 
pela necessidade de se obter maior eficiência econômica e financeira (Browm \& Kimbrough, 2011; Miranda, Vasconcelos, De Luca \& Cabral, 2015; Santos, Vasconcelos \& De Luca, 2013).

Ademais, a internacionalização induz o uso da inovação e proporciona melhores resultados competitivos (Boehe, Larentis, Toni \& Mattia, 2011; Castro, 2011; Costa, Lages \& Hortinha, 2015), e, assim como a inovação, ativos estratégicos e/ou intangíveis, pode facilitar a inserção em mercados estrangeiros (Gomes, Kruglianskas \& Scherer, 2011; Santos et al., 2013), propiciando a abertura de caminhos para atuação em diferentes ambientes (Muzychenko \& Liesch, 2015) em termos de recursos, níveis de concorrência, ambiente jurídico-legal e instituições (Fernandes, Bandeira-De-Mello \& Zanni, 2012). Essa temática fundamenta-se na Visão Baseada em Recursos (VBR), segundo a qual a competitividade empresarial emerge dos diferenciais competitivos conferidos por seus fatores externos, assim como pelos recursos internos disponíveis ou gerados pela organização. Nesse sentido, a internacionalização é vista como um mecanismo de inclusão da empresa no mercado estrangeiro (Andersen, 1997), enquanto a inovação também é reconhecida como um recurso estratégico de grande representatividade (Barney, 1991; Wernerfelt, 1984), refletindo fortemente no poder de competitividade da companhia (Cheng, Yang \& Sheu, 2014; Wang, 2014), como defende a VBR.

Com base no exposto, e devido à deferência que a temática denota nas pesquisas e no ambiente empresarial, eis que surge a pergunta norteadora deste estudo: Qual a influência da internacionalização e da inovação na competitividade das empresas, sob o enfoque da VBR? Nessa perspectiva, o estudo tem como objetivo geral investigar a influência da internacionalização e da inovação na competitividade em empresas de países do BRICS (Brasil, Rússia, Índia, China e África do Sul) listadas na Bolsa de Valores de Nova lorque (The New York Stock Exchange - NYSE), sob a perspectiva da VBR.

Constituem objetivos específicos do estudo: i) identificar semelhanças e diferenças na configuração da competitividade nas empresas mais internacionalizadas e mais inovadoras em comparação com aquelas menos internacionalizadas e menos inovadoras; e ii) investigar a correlação entre internacionalização e inovação nas organizações.

O estudo se justifica pela relevância dos temas internacionalização (Boehe et al., 2011; Stal, 2010) e inovação (Santos et al., 2013; Tavassoli \& Karlsson, 2015) e sua relação mais precisa com a competitividade nas companhias. Apesar de individualmente a internacionalização e a inovação serem temas amplamente discutidos na literatura, é releante que sejam explorados na perspectiva da competitividade empresarial, uma vez que são essenciais para o fortalecimento da economia (Ribeiro, 2016) e apontados como de grande valor organizacional (Brito \& Brito, 2012). Outrossim, poucas pesquisas analisaram empiricamente a competitividade tendo como proxy o índice de Herfindahl-Hirschman (Albuquerque Filho, Macêdo, Moura, Fank, \& Heberle, 2018; Giroud \& Mueller, 2010; Moura et al., 2013).

Além disso, considera empresas de capital aberto localizadas em países do BRICS, que, por serem emergentes com possibilidade de ascensão (Mazzioni, Rigo, Klann \& Silva Junior, 2014), apresentam considerável representatividade para investidores e acadêmicos (Aguilera, Ciravegna, Cuervo-Cazurra \& Gonzalez-Perez, 2017) , tendo em vista ainda, que a internacionalização de empresas de economias emergentes se comporta de maneira distinta das empresas de economias desenvolvidas (Andrade \& Galina, 2013).

Destaca-se também a contribuição do estudo para - campo acadêmico ao ponderar a internacionalização e a inovação como métricas multidimensionais para análise do envolvimento internacional das empresas e da adaptação estratégica de recursos como fontes de vantagem competitiva.

\section{FUNDAMENTAÇÃO TEÓRICA}

\section{Internacionalização, inovação e competitividade}

A VBR enfatiza que a competitividade empresarial emerge dos diferenciais competitivos conferidos pelos recursos externos e internos disponíveis ou gerados pela organização (Barney, 1991). Nesse contexto, as vantagens competitivas exigidas pelo mercado empresarial estimulam a empresa a buscar alternativas que contribuam para a ampliação de seu poder de competitividade (Dai, Maksimov, Gilbert \& Fernhaber, 2014; Knight \& Liesch, 2016), sendo a internacionalização uma das principais estratégias 
Influência da Internacionalização e da Inovação na Competitividade Empresarial

utilizadas para o alcance dessas vantagens (Zen, 2012).

A internacionalização configura-se como um processo organizacional que proporciona crescimento e diversificação de investimentos (Brock \& Yaffe, 2008) à empresa que pretende inserir-se no mercado externo (Machado \& Carvalho, 2013), a fim de adquirir novas oportunidades em ambientes emergentes que possuem sistema jurídico-legal e cultura distintos daqueles de seu país (Floriani \& Fleury, 2012).

Em outros termos, a internacionalização é tida como o processo em que a empresa almeja deixar de operar apenas no mercado doméstico, e passa a vislumbrar mercados estrangeiros em busca de oportunidades para ofertar produtos e serviços capazes de suprir as necessidades dos consumidores (Javalgi, Griffith \& White, 2003; Sharma \& Erramilli, 2004).

Floriani e Fleury (2012) e Santos, Vasconcelos e De Luca (2015) destacam que a internacionalização pode influenciar de forma significativa as estruturas da empresa, em decorrência das imposições competitivas do mercado internacional, que se diferem daquelas do país de origem, já que compreendem a adaptação estratégica de recursos e estrutura, além do envolvimento internacional.

A VBR fundamenta a internacionalização por meio da apropriação de recursos de difícil replicação, aquisição ou substituição que gerem alguma vantagem competitiva para a organização (Barney, 1991; Knight \& Cavusgil, 2004; Stal, 2010). Nesse sentido, a internacionalização é vista como uma estratégia por meio da qual os gestores vislumbram oportunidades além-fronteiras com a intenção de ampliar seus recursos, no intuito de aplicá-los em outros países, objetivando maximizar seu retorno (Jung \& Bansal, 2009).

Carneiro e Dib (2007) destacam o aspecto multidimensional da internacionalização, frisando que as motivações que levam a empresa a se internacionalizar são bem mais complexas do que a mera busca por novos mercados ou de regiões com baixo custo de insumos, fuga de barreiras comerciais ou aproveitamento de incentivos governamentais estrangeiros, incluindo, nesse aspecto multidimensional, a internacionalização quanto ao capital estrangeiro (Maia, Vasconcelos \& De Luca, 2013; Santos et al., 2013), receitas de exportação
(Floriani \& Fleury, 2012) e mercados externos (Mitter, Duller, Feldbauer-Durstmüller \& Kraus, 2014; Platchek; Floriani; Borini, 2012).

Para pôr em prática suas estratégias, e dessa forma estimular a ascensão de sua competitividade, a empresa deve realizar investimentos que na maioria das vezes são insuficientes para cobrir os custos de sua realização, sendo indispensável a procura de recursos externos que viabilizem a implementação de suas estratégias, por meio da internacionalização (Bartoloni, 2013).

Outra estratégia competitiva que merece destaque é a inovação, tida como a combinação ou introdução de bens, serviços, métodos de produção, mercados ou negócios (Schumpeter, 1988) de forma nova ou otimizada das práticas organizacionais (Muzychenko \& Liesch, 2015; Prajogo, 2016; Porter, 1996).

Segundo Arantes e Furquim (2011), Lopes, Beuren e Dametto (2016) e Teh, Kayo e Kimura (2008), a inovação tem ligação direta com o aumento da competitividade, refletindo em maior desenvolvimento da organização. Barney (1996), Benetti et al. (2014) e Lopes et al. (2016) apontam a inovação como um importante instrumento de vantagem competitiva, de maneira que possibilita o desenvolvimento de novas capacidades e recursos para aumentar a produtividade.

As estratégias de inovação atuam como fatores relevantes de implementação de ideias que refletem na geração de valor para os clientes (Hurley \& Hult, 1998), sendo explicadas pela VBR na medida em que se tornam representativas dentro da empresa e induzem a melhoria do desempenho (Barney, 1991; Wernerfelt, 1984), atrelado ao valor competitivo (Cheng et al., 2014; Terziovski, 2010; Wang, 2014).

A definição de estratégias competitivas ligadas aos investimentos direcionados para inovação impacta o processo decisório (Brock \& Yaffe, 2008), de maneira que a inovação atua como um importante esquema estratégico de proteção e vantagem de mercado (Jayaram, Oke \& Prajogo, 2014; Porter, 1996; Prajogo, 2016), e contribui para o aumento do poder de competitividade (Lopes et al., 2016).

A competência em organizar os recursos estratégicos de forma inovadora e tomar decisões com vistas à otimização dos recursos (Schreiber, Bessi, Puffal \& Tondolo, 2013) e o envolvimento em mercados estrangeiros, como a oferta de produtos e 
serviços (Sharma \& Erramilli, 2004), propiciam às organizações assumir posições mais competitivas no mercado, conforme preceitos da VBR (Teece, Pisano \& Shuen, 1997).

Dentre os estudos empíricos sobre os construtos internacionalização e inovação e a competitividade, destaca-se o de Ribeiro (2016), que estudou a relação da internacionalização e da inovação em cinco empresas brasileiras, constatando um ciclo vicioso de interdependência; assim, a inovação possibilitou e influenciou a internacionalização das companhias que, por meio desse processo e pela concorrência do mercado, sentiu a necessidade de inovar, enfatizando também que a existência de tais elementos proporciona o aumento da competitividade.

De Paula, Ferreira e Quinte (2019) identificaram os fatores planejamento, inovação e qualificação profissional como base para uma estrutura apta a enfrentar o mercado internacional, contribuindo para o aumento da competitividade empresarial. Conto, Antunes e Vaccaro (2016) também identificaram a inovação como fator que contribui para a conquista de vantagens competitivas em uma firma produtora de suco e vinho orgânicos.

Empiricamente, Alvarez e Marin (2013), em estudo sobre a ação combinada de empresas multinacionais (internacionalização) e a capacidade de absorção e criação de tecnologia (inovação) em países em desenvolvimento com sofisticados mercados de alta tecnologia, identificaram que existe uma relação positiva e significativa dos construtos em relação à competitividade das empresas. Tais resultados são semelhantes aos evidenciados por Pangarkar, (2008) e Tarpczynski e Wrona (2013) no que se refere à internacionalização, como estratégia que reflete positivamente sobre a competitividade das empresas. Ademais, observa-se uma associação positiva entre a inovação e a competitividade, proporcionando maior lucratividade para a organização e maior valor para os diversos stakeholders (Botelho \& Guissoni, 2016). Conforme ponderado por Prajogo (2016), ocorre uma relação estreita entre inovação e competitividade, sendo reconhecida como uma estratégia eficaz na conquista de novos mercados.

Assim, com base nos fundamentos da VBR, em que a competitividade depende de fatores externos, como o mercado internacional, além de fatores internos que promovem a adaptação estratégica e a otimização de recursos, como a criação e a absorção de novas tecnologias, propõem-se as seguintes hipóteses:

$\mathrm{H}_{1}$ - a internacionalização influencia a competitividade nas empresas; e

$\mathrm{H}_{2}-\mathrm{a}$ inovação influencia a competitividade nas empresas.

\section{PROCEDIMENTOS METODOLÓGICOS}

A população da pesquisa reuniu 90 empresas não financeiras de países do BRICS listadas na Nyse em 26 de março de 2018. Na composição da amostra, foram consideradas somente empresas que detinham as informações necessárias para a construção das variáveis, compreendendo os exercícios financeiros de 2015, 2016 e 2017. Em decorrência disso, foram excluídas 26 companhias, resultando, portanto, em uma amostra de 64 empresas.

A delimitação do espaço temporal se justifica em função das estratégias competitivas. No que tange à inovação, o Manual de Oslo (OCDE, 2005) orienta que a dimensão do período de observação para as pesquisas não exceda três anos nem seja inferior a um ano.

Na Tabela 1, observam-se a descrição, as fontes e o suporte teórico das variáveis utilizadas na pesquisa. 
Tabela 1

Variáveis utilizadas na pesquisa

\begin{tabular}{|c|c|c|c|}
\hline Variável & Descrição & Fonte & Suporte teórico \\
\hline \multicolumn{4}{|c|}{ Variável Dependente } \\
\hline $\begin{array}{l}\text { Competitividade } \\
\text { (COMPET) }\end{array}$ & $\begin{array}{l}\text { Índice de Herfindahl- } \\
\text { Hirschman }\end{array}$ & $\begin{array}{l}\text { Thomson Reuters } \\
\text { Worldscope }{ }^{\circledR}\end{array}$ & $\begin{array}{l}\text { Dhaliwal, Gleason, Heitzman e } \\
\text { Melendrez (2008) } \\
\text { Giroud e Mueller (2010) } \\
\text { Moura } \text { et al. }(2013)\end{array}$ \\
\hline \multicolumn{4}{|l|}{ Variáveis Independentes } \\
\hline \multirow[t]{2}{*}{ Internacionalização } & $\begin{array}{l}\text { (LISTAG) - Número de } \\
\text { bolsas de valores em que a } \\
\text { empresa é listada }\end{array}$ & Formulário 20-F & $\begin{array}{l}\text { Hassel et al. (2003) } \\
\text { Santos et al. (2013) }\end{array}$ \\
\hline & $\begin{array}{l}\text { (QPAIS })- \text { Número de } \\
\text { países onde a empresa } \\
\text { possui unidades instaladas }\end{array}$ & Formulário $20-\mathrm{F}$ & $\begin{array}{l}\text { Borini, Floriani e Fleury (2012) } \\
\text { Ruigrok e Wagner (2003) } \\
\text { Santos, Vasconcelos e De Luca (2015) }\end{array}$ \\
\hline \multirow{2}{*}{ Inovação } & $\begin{array}{l}\text { Patentes (INOVPAT) - } \\
\text { Dummy } \\
\text { Se a empresa possui } \\
\text { patentes }=1 \\
\text { Caso contrário }=0\end{array}$ & Formulário $20-\mathrm{F}$ & $\begin{array}{l}\text { Kayo et al. }(2006) \\
\text { Moura e Galina (2009) } \\
\text { Teh } \text { et al. }(2008)\end{array}$ \\
\hline & $\begin{array}{l}\text { Índice de inovação } \\
\text { (INOVPD) - Razão entre } \\
\text { o valor do dispêndio com } \\
\text { P\&D e o da receita líquida } \\
\text { de vendas }\end{array}$ & Formulário $20-\mathrm{F}$ & $\begin{array}{l}\text { Blazsek e Escribano (2016) } \\
\text { Nekhili, Boubaker e Lakhal (2012) }\end{array}$ \\
\hline \multicolumn{4}{|c|}{ Variáveis de Controle Organizacional } \\
\hline $\begin{array}{l}\text { Tamanho } \\
\text { (TAM) }\end{array}$ & $\begin{array}{l}\text { Logaritmo Natural do } \\
\text { valor do Ativo }\end{array}$ & $\begin{array}{l}\text { Thomson Reuters } \\
\text { Worldscope } ®\end{array}$ & $\begin{array}{l}\text { Badolato, Donelson e Ege (2014) } \\
\text { Chi et al. (2015) } \\
\text { Huguet e Gandía (2016) }\end{array}$ \\
\hline $\begin{array}{l}\text { Rentabilidade } \\
\text { (ROE) }\end{array}$ & $\begin{array}{l}\text { Razão entre o valor do } \\
\text { Lucro Líquido e o do } \\
\text { Patrimônio Líquido }\end{array}$ & $\begin{array}{l}\text { Thomson Reuters } \\
\text { Worldscope }{ }^{\circledR}\end{array}$ & $\begin{array}{l}\text { Geiger e Makri (2006) } \\
\text { Gonçalves Filho, Veit e Monteiro } \\
(2013) \\
\text { Lima e Carmona (2010) }\end{array}$ \\
\hline $\begin{array}{l}\text { Endividamento } \\
\text { (END) }\end{array}$ & $\begin{array}{l}\text { Proporção do valor do } \\
\text { Passivo em relação ao } \\
\text { valor do Ativo }\end{array}$ & $\begin{array}{l}\text { Thomson Reuters } \\
\text { Worldscope } ®\end{array}$ & $\begin{array}{l}\text { Kayo et al. (2006) } \\
\text { O’ Brien }(2003)\end{array}$ \\
\hline $\begin{array}{l}\text { Crescimento } \\
\text { (CRESC) }\end{array}$ & $\begin{array}{l}\text { Proporção do valor da } \\
\text { Receita Operacional no } \\
\text { ano } t \text { em relação ao do ano } \\
t-1\end{array}$ & $\begin{array}{l}\text { Thomson Reuters } \\
\text { Worldscope } ®\end{array}$ & $\begin{array}{l}\text { Brito, Brito e Vasconcelos (2007) } \\
\text { Brito e Vasconcelos (2009) }\end{array}$ \\
\hline \multicolumn{4}{|c|}{ Variáveis de Controle Institucional } \\
\hline $\begin{array}{l}\text { Sistema Jurídico-legal } \\
\text { (SISLEG) }\end{array}$ & $\begin{array}{l}\text { Dummy } \\
\text { Empresa sediada em país } \\
\text { com sistema Commom } \\
\text { Law ou } \\
\text { Civil Law }=1 \\
\text { Caso contrário }=0\end{array}$ & Juriglobe & $\begin{array}{l}\text { Bushman e Piotroski (2006) } \\
\text { Carmo, Ribeiro e Carvalho (2011) } \\
\text { La Porta, Lopez-De-Silanes, Shleifer } \\
\text { e Vishny (1998). }\end{array}$ \\
\hline $\begin{array}{l}\text { Localização Regional } \\
\text { (LOCREG) }\end{array}$ & $\begin{array}{l}\text { Dummy } \\
\text { Empresa sediada em país } \\
\text { localizado na região Leste } \\
=1 \\
\text { Caso contrário }=0\end{array}$ & Mapa-múndi & $\begin{array}{l}\text { Gelfan, Erez e Aycan (2007) } \\
\text { House } \text { et al. }(2004) \\
\text { Kull e Wacker (2010) }\end{array}$ \\
\hline
\end{tabular}

Fonte: Elaborada pelos autores.

Em relação à competitividade, conforme apresentado na Tabela 1, foi utilizado o Índice de Herfindahl-Hirschman (HHI), calculado a partir da Equação 1, em que ßi representa a proporção do valor do Ativo da empresa $i$ em relação ao valor total da amostra. 


$$
H H I=\sum_{i=1}^{n} \beta_{i}^{2}
$$

Para resolução da Equação 1, optou-se por utilizar valores de Ativo, assim como procederam Moura et al. (2013), pois, segundo eles, no decorrer dos períodos o valor do Ativo tem menor oscilação do que o de qualquer outra variável, como, por exemplo, a Receita Operacional.

Ainda na Tabela 1, percebe-se ainda que a variável internacionalização é analisada por meio do Logaritmo Natural do número de bolsas de valores em que a empresa é listada e do número de países onde ela possui unidades instaladas, ambos considerados proxies para internacionalização, coletados no website da Securities and Exchange Commission (SEC), por meio dos formulários 20-F referentes aos três exercícios financeiros em análise. As informações para a variável inovação - pesquisa e desenvolvimento (P\&D) e registro de patentes também foram coletadas dos formulários 20-F.

Alinhado à literatura especializada, o estudo adotou como variáveis de controle organizacional o tamanho da empresa, representado pelo Logaritmo Natural do valor do Ativo (Huguet \& Gandía, 2016); a rentabilidade, mensurada pelo Retorno Sobre o Patrimônio Líquido (ROE), obtido mediante divisão do valor do Lucro Líquido pelo do Patrimônio Líquido (Lima \& Carmona, 2010); o índice de endividamento, calculado mediante divisão do valor do Passivo (Circulante + Não Circulante) pelo do Ativo (Circulante + Não Circulante) (Kayo et al., 2006); e o crescimento, mensurado pela divisão do valor da Receita Operacional no ano $t$ pelo do ano $t-1$ (Brito \& Vasconcelos, 2009).

Além disso, e considerando-se que a amostra contempla empresas de países distintos, foram utilizadas as seguintes variáveis de controle institucional: o sistema jurídico-legal, representado por uma dummy que recebeu o valor " 1 " quando a empresa se localiza em país que adota o sistema Common Law ou o Civil Law, e o valor " 0 " no caso contrário (Carmo, Ribeiro \& Carvalho, 2011); e a localização regional, também representada por uma dummy que recebeu o valor " 1 " quando a empresa é sediada em país localizado na Região

(Equação 1)

Leste, e o valor " 0 " no caso contrário (House, Hanges, Javidan, Dorfman \& Gupta, 2004).

O tratamento e a análise dos dados são guiados pelos objetivos delineados no estudo. Para se atingir o primeiro objetivo específico - identificar semelhanças e diferenças na configuração da competitividade nas empresas mais internacionalizadas e mais inovadoras em comparação com aquelas menos internacionalizadas e menos inovadoras -, realizou-se o teste de diferenças entre médias ( $t$ de Student), já que, por meio do teste de normalidade de KolmogorovSmirnov, a distribuição das variáveis mostrou-se normal, ao nível de 5\% (Fávero \& Belfiore, 2017). Dessa forma, foram criados dois grupos para representação das empresas: o primeiro (G1) reúne as empresas mais internacionalizadas ou mais inovadoras, ou seja, cuja variável analisada assinala valores acima ou igual aos valores da mediana; e o segundo (G2) reúne as empresas menos internacionalizadas ou menos inovadoras, isto é, cuja variável analisada registra valores abaixo da mediana (Oliveira, Thomas \& Espadanal, 2014).

Para se atingir o segundo objetivo específico investigar a correlação entre internacionalização e inovação nas organizações -, dado como válido o pressuposto da normalidade amostral, utilizou-se o teste de correlação de Pearson.

Com o propósito de atender ao objetivo geral e testar as duas hipóteses formuladas, realizou-se a análise de regressão linear múltipla com dados em painel, para se inferir acerca da influência das estratégias de internacionalização e das estratégias de inovação na competitividade das organizações. Os dados foram organizados em painéis não balanceados, levando-se em conta uma amostra com 64 companhias, para um período de três anos.

Os modelos econométricos do estudo estão apresentados nas equações 2 , 3 e 4 . 


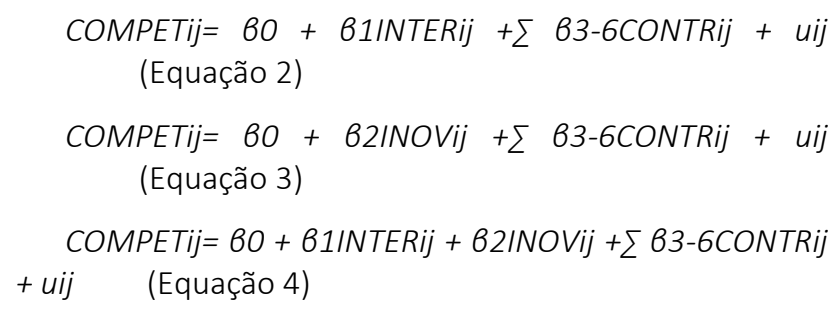

Em que:

COMPET representa a competitividade;

INTER exprime a internacionalização, representada pelas variáveis LISTAG e QPAIS;

INOVij corresponde a inovação, retratada pelas patentes (INOVPAT) e pelo Índice de P\&D (INOVPD);

CONTRij representa as variáveis de controle do modelo econométrico, relativas à empresa $i$ no período $j$, representadas por tamanho (TAM), rentabilidade (ROE), endividamento (END), crescimento (CRESC), sistema jurídico-legal (SISLEG) e localização regional (LOCREG);
BO é o intercepto; e

uij é o termo de erro estocástico da regressão linear múltipla com dados em painel.

Destaque-se que foram realizados os testes $F$ de Chow, LM de Breusch-Pagan e de Hausman para se identificar o modelo de estimação mais adequado entre POLS, Efeitos Fixos e Efeitos Aleatórios. De acordo com o teste $\mathrm{F}$ de Chow (sig. $<0,05$ ), o teste LM de BreuschPagan (sig.<0,05) e o teste de Hausman (sig.>0,05), a estimação por efeitos aleatórios caracteriza-se como a mais adequada para a análise da regressão. Além disso, aplicou-se a técnica robust, porquanto capaz de corrigir eventuais problemas estatísticos quanto à existência de heterocedasticidade (Fávero \& Belfiore, 2017).

\section{ANÁLISE DOS RESULTADOS}

A análise dos resultados tem início com a apresentação da estatística descritiva das variáveis do estudo (Tabela 2), que reflete as características das empresas da amostra.

Tabela 2

Estatística descritiva

\begin{tabular}{c|c|c|c|c|c|c|c|c}
\hline & \multicolumn{7}{|c}{ Variável } \\
\cline { 2 - 8 } Estatística Descritiva & COMPET & INOVPD & LISTAG & QPAIS & TAM & ROE & END & CRESC \\
\hline № de Observações & 192 & 102 & 171 & 170 & 177 & 176 & 177 & 189 \\
Média & 0,003 & 3,136 & 0,556 & 0,781 & 8,580 & $-0,244$ & 0,509 & 1,003 \\
Desvio-padrão & 0,029 & 26,750 & 0,622 & 0,697 & 2,062 & 9,003 & 0,271 & 0,957 \\
Coeficiente de. & 9,666 & 8,529 & 1,118 & 0,892 & 0,240 & 36,897 & 0,532 & 0,954 \\
Variação & 0,000 & 0,000 & 0,000 & 0,000 & 4,715 & $-89,148$ & 0,000 & 0,000 \\
Mínimo & 0,403 & 269,377 & 3,218 & 3,218 & 14,872 & 65,090 & 1,853 & 12,595 \\
Máximo &
\end{tabular}

Fonte: Elaborada pelos autores.

Na Tabela 2, observa-se que a variável tamanho é a que mais se destacou, em decorrência de sua média $(8,580)$, amplitude $(10,157)$ e homogeneidade $(0,240)$. Contudo, foi a que apresentou maior disparidade, revelando uma elevada dispersão dos valores do Ativo registrados pelas organizações.

É notável que das variáveis de internacionalização, o número de países em que a empresa possui unidades instaladas (QPAIS) foi a que apresentou maior média $(0,781)$ e maior homogeneidade $(0,892)$. Em contrapartida, foi a que assinalou maior dispersão
$(0,697)$ e igual amplitude $(3,218)$, quando comparada com o número de bolsas em que a empresa é listada (LISTAG).

As empresas com maior número de unidades instaladas no exterior, no período analisado, foram: 500.Com Ltd, Aluminum Corp Of China Ltd, Ambev S/A, Cheetah Mobile Inc., China New Borun Corp., Ehi Car Services Ltd, Phoenix New Media Ltd e Wns (Holdings) Ltd. As empresas listadas em maior número de bolsas de valores foram: Aluminum Corp. of China Ltd, Ambev S/A, Anglogold Ashanti Ltd, China Petroleum \& Chemical 
Corp, Cnooc Ltd, Infosys Ltd, Mix Telematics Ltd, Mobile Telesystems Pjsc, Petrochina Co Ltd e Wipro Ltd.

Verifica-se ainda, pela Tabela 2, que a variável inovação (INOVPD) apresentou maior amplitude, maior dispersão e maior heterogeneidade. As companhias que registraram os maiores valores em P\&D foram: 500.Com Ltd, Daqo New Energy Corp., China Digital Tv Holding Co. Ltd, Phoenix New Media Ltd, Renren Inc., Sasol Ltd e Wipro Ltd.

Na sequência, e no intuito de se identificar semelhanças e diferenças na configuração da competitividade no que tange a internacionalização
(LISTAG e QPAIS) e inovação (INOVPD), são apresentados os resultados dos testes de diferenças entre médias. Vale lembrar que, como exposto no tópico da metodologia, para aplicação dos testes, as empresas foram classificadas em dois grupos: G1, que engloba as empresas mais internacionalizadas ou mais inovadoras; e G2, que reúne as menos internacionalizadas ou menos inovadoras.

A Tabela 3 apresenta o resultado dos testes de diferenças entre médias da competitividade no tocante a internacionalização.

Tabela 3

Teste de diferenças entre médias da competitividade pela perspectiva da internacionalização

\begin{tabular}{|c|c|c|c|c|c|c|}
\hline \multirow{2}{*}{ Variável } & \multirow{2}{*}{ COMPET } & \multirow{2}{*}{$\mathrm{N}$} & \multicolumn{2}{|c|}{ Teste $t$ de Student } & \multicolumn{2}{|c|}{ Teste de Levene } \\
\hline & & & Média & Sig. & $\mathrm{F}$ & Sig. \\
\hline \multirow[b]{2}{*}{ LISTAG } & G1 & 39 & 0,0028430495 & 0,878 & \multirow{2}{*}{0,122} & \multirow{2}{*}{0,727} \\
\hline & $\mathrm{G} 2$ & 132 & 0,0037196576 & 0,795 & & \\
\hline \multirow{2}{*}{ QPAIS } & G1 & 56 & 0,0002268586 & 0,073 & \multirow{2}{*}{11,359} & \multirow{2}{*}{$0,001^{(*)}$} \\
\hline & $\mathrm{G} 2$ & 114 & 0,0016226005 & $0,013^{(* *)}$ & & \\
\hline
\end{tabular}

Nota. $\left({ }^{*}\right)$ significante ao nível de $1 \% ;(* *)$ significante ao nível de $5 \%$.

Fonte: Elaborada pelos autores.

Com base nos dados da Tabela 3, pode-se afirmar que, pelo teste de Levene, o número de bolsas de valores em que a empresa é listada (LISTAG) apresenta variabilidade semelhante em termos de competitividade tanto para as empresas mais internacionalizadas como para as menos internacionalizadas. Além disso, destaca-se que a internacionalização mostra-se, em geral, igual em ambos os grupos ao considerar seus níveis de competitividade, já que o teste $t$ de Student não apresentou significância estatística.

Por outro lado, o número de países onde a empresa possui unidades instaladas apontou diferenças estatisticamente significantes na variabilidade e na média, em relação à competitividade, pontuando que as empresas com maior dispersão geográfica possuem menor média $(0,0002268586)$ em termos de competitividade, quando comparadas com as empresas presentes em menor número de países. Tal resultado destoa dos achados de Boermans e Roelfsema (2012) e Shearmur, Doloreux e Laperrière (2015), segundo os quais a abertura de mercados externos confere maior potencial de expansão e crescimento da empresa, atuando como mecanismo de retroalimentação de sua competitividade.

De forma similar à análise das variáveis de internacionalização, a Tabela 4 mostra os resultados do teste de diferenças entre médias para o construto inovação, particularmente para a variável INOVPD, já que INOVPAT é uma variável categórica.

Tabela 4

Teste de médias da competitividade pela perspectiva da inovação

\begin{tabular}{c|c|c|c|c|c|c}
\hline \multirow{2}{*}{ Variável } & \multirow{2}{*}{ COMPET } & \multirow{2}{*}{$\mathbf{N}$} & \multicolumn{2}{|c}{ Teste $t$ de Student } & \multicolumn{2}{c}{ Teste de Levene } \\
\cline { 3 - 7 } & \multirow{2}{*}{ INOVPD } & & Média & Sig. & F & Sig. \\
\cline { 2 - 5 } & G1 & 51 & 0,0006870349 & 0,044 & 12,121 & $0,001^{(*)}$ \\
\hline
\end{tabular}

Nota. $\left({ }^{*}\right)$ significante ao nível de $1 \% ;\left({ }^{*}\right)$ significante ao nível de $5 \%$.

Fonte: Elaborada pelos autores. 
Com base na Tabela 4, depreende-se que a inovação, medida pelo nível de P\&D, apresenta variabilidade e médias diferentes em termos de competitividade entre os dois grupos.

Os resultados sugerem uma alta heterogeneidade entre os níveis de competitividade evidenciados por $\mathrm{G} 1$ e G2 (teste de Levene, sig. $<0,05$ ), denotando que as empresas mais inovadoras apresentam menor competitividade $(0,06 \%)$ em relação às empresas menos inovadoras, que tendem a apresentar maior competitividade $(0,3 \%)$, contrariando Botelho e Guissoni (2016), segundo os quais as empresas inovadoras tendem a apresentar maior competitividade.

Posteriormente, e para se investigar a correlação entre internacionalização e inovação, utilizou-se a correlação de Pearson, cujos resultados são mostrados na Tabela 5.

Tabela 5

Correlação de Pearson

\begin{tabular}{c|c|c|c|c|c|c|c|c}
\hline Variável & COMPET & INOVPD & LISTAG & QPAIS & TAM & ROE & END & CRESC \\
\hline COMPET & 1,000 & & & & & & & \\
\hline INOVPD & $-0,037$ & 1,000 & & & & & & \\
\hline LISTAG & 0,033 & $-0,109$ & 1,000 & & & & & \\
\hline QPAIS & $-0,106$ & 0,050 & $-0,024$ & 1,000 & & & & \\
\hline TAM & $0,296^{(*)}$ & $-0,167$ & $-0,291^{(*)}$ & 0,017 & 1,000 & & & \\
\hline ROE & 0,006 & 0,012 & $-0,114$ & $-0,045$ & $-0,006$ & 1,000 & & \\
\hline END & $\left.-0,157^{* *}\right)$ & $-0,155$ & $-0,029$ & 0,028 & 0,005 & 0,001 & 1,000 & \\
\hline CRESC & 0,028 & 0,056 & $-0,025$ & 0,019 & $-0,137$ & $0,160^{(* *)}$ & 0,028 & 1,000 \\
\hline
\end{tabular}

Nota. $\left({ }^{*}\right)$ significante ao nível de $1 \% ;\left({ }^{* *}\right)$ significante ao nível de $5 \%$.

Fonte: Elaborada pelos autores.

A partir dos dados da Tabela 5, observa-se que as variáveis de internacionalização (LISTAG e QPAIS) não se correlacionam com a variável de inovação INOVPD, contrariando os resultados encontrados por Boehe et al. (2011), Castro (2011), Costa et al. (2015), Gomes et al. (2011) e Halilem, Amara e Landry (2014), que identificaram indícios de correlação entre os construtos. Assemelham-se, porém, aos achados de Reynoso e Figueroa (2010), segundo os quais, mesmo as empresas não enquadradas como inovadoras podem se encontrar em acelerado processo de internacionalização, simplesmente porque possuem o controle dos seus ativos estratégicos. De forma adicional, nota-se também que as variáveis de interesse não apresentaram correlações estatisticamente significantes com a competitividade, dissociando-se dos indícios encontrados por Cheng et al. (2014) e Wang (2014).

Saliente-se, contudo, que a variável tamanho se correlaciona positivamente com o nível de competitividade, sugerindo que, na medida em que a empresa se expande, sua competitividade cresce na mesma proporção, confirmando os apontamentos de Cho e Pucik (2005) e Terra, Barbosa e Bouzada (2015). Por outro lado, o tamanho se correlaciona negativamente com o número de bolsas de valores em que a empresa é listada.

Percebe-se também que quanto menor for o endividamento da empresa, maior será sua competitividade, ou seja, existe uma associação negativa entre as duas variáveis, como constatado por O’Brien (2003). Além disso, é possível afirmar que níveis de crescimento mais altos e mais baixos impactam positivamente a rentabilidade da organização, ou seja, o crescimento agrega valor a si mesma utilizando seus próprios recursos.

Para se alcançar o objetivo geral e testar as hipóteses, realizou-se a análise de regressão linear múltipla com dados em painel. A Tabela 6 mostra os resultados da análise para verificar a influência da internacionalização (LISTAG e QPAIS) e da inovação (INOVPT e INOVPAT) na competitividade das organizações. 
Tabela 6

Análise de regressão

\begin{tabular}{|c|c|c|c|c|c|c|}
\hline $\begin{array}{c}\text { Modelo } \\
\text { (COMPET) }\end{array}$ & \multicolumn{2}{|c|}{$\begin{array}{l}\text { INTERNACIONALI- } \\
\text { ZAÇÃO } \\
(1) \\
\end{array}$} & \multicolumn{2}{|c|}{$\begin{array}{l}\text { INOVAÇÃO } \\
(2)\end{array}$} & \multicolumn{2}{|c|}{$\begin{array}{c}\text { MODELO GLOBAL } \\
\text { (3) }\end{array}$} \\
\hline Estimação & \multicolumn{6}{|c|}{ Efeitos Aleatórios } \\
\hline Variáveis & Coef. & Sig. & Coef. & Sig. & Coef. & Sig. \\
\hline LISTAG & 0,0001 & $(0,750)$ & & & 0,0006 & $(0,561)$ \\
\hline QPAIS & $-0,0009$ & $(0,377)$ & & & $-0,0018$ & $(0,336)$ \\
\hline INOVPAT & & & 0,0004 & $(0,605)$ & 0,0009 & $(0,448)$ \\
\hline INOVPD & & & 0,0000 & $(0,373)$ & 0,0000 & $(0,249)$ \\
\hline TAM & 0,0011 & $(0,001)^{(*)}$ & 0,0013 & $(0,005)^{(*)}$ & 0,0013 & $(0,001)^{(*)}$ \\
\hline ROE & $-0,0000$ & $(0,405)$ & $-0,0000$ & $(0,025)^{* *)}$ & 0,0000 & $(0,596)$ \\
\hline END & $-0,0014$ & $(0,200)$ & $-0,0018$ & $(0,305)$ & $-0,0017$ & $(0,356)$ \\
\hline CRESC & $-0,0000$ & $(0,621)$ & $-0,0000$ & $(0,876)$ & 0,0000 & $(0,807)$ \\
\hline SISLEG & 0,0029 & $(0,363)$ & 0,0016 & $(0,516)$ & 0,0033 & $(0,362)$ \\
\hline LOCREG & 0,0045 & $(0,181)$ & 0,0036 & $(0,180)$ & 0,0061 & $(0,175)$ \\
\hline Intercepto & $-0,0114$ & $(0,028)^{(* *)}$ & $-0,0130$ & $(0,057)^{(* * *)}$ & $-0,0150$ & $(0,035)^{(* *)}$ \\
\hline $\mathrm{N}^{\circ}$ de empresas & \multicolumn{2}{|c|}{64} & \multicolumn{2}{|c|}{64} & \multicolumn{2}{|c|}{64} \\
\hline $\begin{array}{c}\mathrm{N}^{\circ} \text { de } \\
\text { observações }\end{array}$ & \multicolumn{2}{|c|}{151} & \multicolumn{2}{|c|}{101} & \multicolumn{2}{|c|}{93} \\
\hline Wald $\mathrm{Chi}^{2}$ & \multicolumn{2}{|c|}{$23,19^{(*)}$} & \multicolumn{2}{|c|}{$19,78^{(* *)}$} & \multicolumn{2}{|c|}{$26,26^{(*)}$} \\
\hline $\mathrm{P}$-value & \multicolumn{2}{|c|}{0,0031} & \multicolumn{2}{|c|}{0,0112} & \multicolumn{2}{|c|}{0,0034} \\
\hline$R^{2}$ & \multicolumn{2}{|c|}{0,4077} & \multicolumn{2}{|c|}{0,3639} & \multicolumn{2}{|c|}{0,4508} \\
\hline
\end{tabular}

Nota. $\left({ }^{*}\right)$ Significante ao nível de $1 \% ;\left({ }^{* *}\right)$ significante ao nível de $5 \% ;(* *)$ significante ao nível de $10 \%$. Fonte: Elaborada pelos autores.

Com base na Tabela 6, nota-se que, pelo teste de Wald $\mathrm{Chi}^{2}$, os três modelos foram significantes, sendo o primeiro e o terceiro significantes a $1 \%$, enquanto o modelo 2 foi significante a 5\%. Percebe-se, também, que o poder explicativo para os modelos 1,2 e 3 corresponde a 40,77\%, 36,39\% e 45,08\%, respectivamente.

Assemelhando-se ao que foi constatado nos testes de diferenças entre médias (Tabela 3 e Tabela 4), os dados da Tabela 6 permitem inferir que nenhum dos indicadores de internacionalização ou de inovação exerce influência sobre a competitividade nas organizações em nenhum dos modelos da regressão, contrariando os achados de Rodrigues, Rechziegel, Bastos, Fiorillo \& Santos (2012), que destacaram a internacionalização como uma alternativa de crescimento para as empresas que gera retornos e amplia a capacidade competitiva. De forma similar, contraria também os resultados encontrados por Knight e Cavusgil (2004) e Stal (2010). Enquanto isso, Bastos, Feldmann e Fouto (2014) e Prajogo (2016) revelam existir uma forte associação entre a capacidade inovadora da empresa e a utilização de seus recursos, aumentando as chances de alavancar a competitividade, o que é também compartilhado por Arantes e Furquim (2011), Boehe et al. (2011) e Teh et al. (2008).

De forma mais analítica, e com base no estudo realizado, os resultados demonstram que a internacionalização e a inovação não influenciam a competitividade em empresas de países do BRICS, divergindo das constatações preconizadas pela VBR, que revela uma correlação direta entre os recursos e as competências ligados à internacionalização e à inovação na competitividade das empresas. Assim, visto que não há influência estatisticamente significante dos construtos na competitividade das empresas da amostra, rejeitam-se as duas hipóteses $\left(\mathrm{H}_{1}\right.$ e $\left.\mathrm{H}_{2}\right)$ do estudo.

A partir desses resultados, pode-se inferir que empresas em economias emergentes (BRICS) são propensas a apresentar menor base de conhecimento em expansão internacional (Guillen, 2000), ambientes institucionais fracos ou inexistentes, ou até mesmo mercados internos muito pequenos (Khanna \& Rivkin, 2001), e, assim, não são capazes de gerar vantagens competitivas de escala.

Observa-se ainda, que a dependência tecnológica, necessária para maior otimização dos recursos, 
favorece as nações mais desenvolvidas. Assim, as empresas em países em desenvolvimento (BRICS) são mais suscetíveis às pressões externas e possuem mais limitações em seu poder de barganha, comprometendo as competências ligadas à inovação (Coronel, Azevedo \& Campos, 2014).

Revela-se também que, assim como identificado na correlação de Pearson (Tabela 4), a regressão não identificou correlações entre os construtos. Entretanto, detecta-se que, dentre as variáveis de controle, o tamanho influencia positivamente o nível de competitividade nas organizações em todos os modelos, corroborando Shefer e Frenkel (2005) e Zemplinerová e Hromádková (2012), que pontuaram ocorrer uma correlação forte e positiva entre o tamanho e a competitividade, já que as empresas maiores detêm mais facilidade de garantir financiamentos para a expansão de suas atividades. Infere-se também, pelo modelo 2, que na presença de estratégias de inovação, a rentabilidade se mostra relevante, denotando que quanto maior for a rentabilidade, menor será a competitividade da organização, destoando dos resultados de Geiger e Makri (2006), segundo os quais uma maior rentabilidade proporciona maior competitividade à empresa.

Outrossim, destaca-se que o sistema jurídico-legal do país em que a empresa é sediada e a sua localização regional não se mostraram significantes em nenhum dos modelos, contrariando Carmo et al. (2011), segundo os quais o sistema jurídico-legal de um país reflete na reputação e, consequentemente, no desempenho e competitividade da empresa, ao passo que Naor, Jones, Bernardes, Goldstein e Schroeder (2014) identificaram que as diferenças culturais entre as regiões leste e oeste existem e podem impactar a eficácia da empresa, e, por conseguinte, afetar sua competitividade.

\section{CONSIDERAÇÕES FINAIS}

Com a finalidade de se investigar a influência da internacionalização e da inovação na competitividade das empresas, foram analisados os dados referentes ao período de 2015 a 2017 de 64 companhias não financeiras de países do BRICS listadas na Nyse.

Para se identificar semelhanças e diferenças na configuração da competitividade nas empresas mais internacionalizadas e mais inovadoras em comparação com aquelas menos internacionalizadas e menos inovadoras, utilizou-se o teste de diferenças entre médias, mediante classificação das firmas da amostra em dois grupos, segundo a intensidade de internacionalização e inovação, assim como o teste de Levene. Em síntese, verificou-se que o número de bolsas de valores em que a empresa é listada mostrou semelhanças em termos de competitividade tanto para as companhias mais internacionalizadas como para as menos internacionalizadas. Os resultados denotam também que as empresas com maior dispersão geográfica em termos de unidades instaladas possuem menor média quando comparadas com as empresas presentes em maior número de países. Ao se considerar o nível de P\&D apresentado pelas companhias, depreende-se que há variabilidade e médias diferentes em termos de competitividade entre os grupos, de modo que as empresas mais inovadoras apresentam menor poder de competitividade em relação às demais.

Os resultados do teste de correlação indicaram que as variáveis de internacionalização não se correlacionam com as de inovação, considerando-se o nível de P\&D. Entretanto, salienta-se que o tamanho da companhia apresentou correlação positiva com o nível de competitividade, mostrando que na medida em que a empresa se expande, cresce o seu poder de competitividade. Enquanto isso, o endividamento apresentou uma associação negativa com a competitividade, apontando que, quanto menor for o endividamento da empresa, maior será a sua competitividade. Os achados refletem ainda que mais altos ou mais baixos níveis de crescimento impactam positivamente a rentabilidade da organização, de maneira que seu crescimento agrega valor a si mesma, utilizando seus próprios recursos.

O objetivo geral foi atendido por meio da aplicação da regressão múltipla com utilização de dados em painel. Corroborando os achados constatados nos testes de diferenças entre médias e correlação de Pearson, os resultados da regressão indicam que os indicadores de internacionalização e os de inovação não exercem influência sobre a competitividade. Ressalte-se, porém, que, na presença de estratégias de inovação, a rentabilidade se mostra relevante, denotando que quanto maior for a rentabilidade da organização, menor será o seu poder de competitividade.

Diante do exposto, e como a internacionalização e a inovação não afetam, em completude, a competitividade nas companhias, as duas hipóteses do estudo foram rejeitadas, notando-se, que a VBR 
não pode ser confirmada quanto a esses elementos nas empresas localizadas nos países da amostra, pois não foram apresentados indícios de correlação entre os construtos. Assim, denota-se que, para se tornar mais competitivas, as empresas sediadas em países do BRICS não necessariamente optam por investir em um número maior de bolsas, instalação de unidades em outros países, como também investimentos relevantes em $P \& D$.

Este estudo contribui, portanto, para uma maior visão da relação entre a internacionalização e a inovação na competividade das organizações, considerando o impacto e as consequências desses elementos nas empresas de capital aberto localizadas em países do BRICS. O estudo revela contribuições relevantes para investidores, reguladores e diversos stakeholders, como um instrumento de conhecimento efetivo do desenvolvimento e fatores competitivos dessas organizações, apontando-se ainda, de grande relevância para maiores esclarecimentos e ampliação da discussão dos temas

\section{REFERÊNCIAS}

Aguilera, R. V., Ciravegna, L., Cuervo-Cazurra, A., \& Gonzalez-Perez, M. A. (2017). Multilatinas and the internationalization of Latin American firms. Journal of World Business, 52(4), 447-460.

Albuquerque Filho, A. R., Macêdo, F. F. R. R., Moura, G. D., Fank, D. R. B., \& Heberle, E. L. (2018). Fatores Determinantes da Intangibilidade em Companhias Abertas Familiares. Revista de Contabilidade do Mestrado em Ciências Contábeis da UERJ, 23(2), 37-52.

Alvarez, I., \& Marin, R. (2013). FDI and technology as levering factors of competitiveness in developing countries. Journal of International Management, 19(3), 232-246.

Andrade, A. M. F., \& Galina, S. V. R. (2013). Efeitos da internacionalização sobre $\mathrm{O}$ desempenho de multinacionais de economias em desenvolvimento. Revista de Administração Contemporânea, 17(2), 239262.

Andersen, O. (1997). Internationalization and market entry mode: A review of theories and conceptual frameworks. MIR: Management International Review, 27-42.

Furquim, N. R., \& Arantes, E. C. (2011). Estratégias de internacionalização e de inovação como diferenciais para a expansão de uma empresa multinacional. Revista Eletrônica de Negócios Internacionais, 6(2), 116-137. no âmbito econômico e acadêmico. Destaca-se ainda, a contribuição do estudo, haja vista que são incipientes as pesquisas acadêmicas abordando os construtos aqui analisados sob a perspectiva da competitividade, em especial, com empresas de países emergentes.

Dentre as limitações enfrentadas neste estudo, destaca-se o pequeno lapso temporal considerado, embora as pesquisas sobre o construto inovação, segundo o Manual de Oslo, devam se restringir ao máximo de três anos; além do reduzido tamanho da amostra, devido à ausência de informações de muitas empresas no relatório 20-F. Assim, para pesquisas futuras, sugere-se: utilizar uma amostra distinta, com companhias listadas em outras bolsas de valores; adotar outras medidas relacionadas à internacionalização e à inovação; e analisar as relações entre a competitividade e outros fatores organizacionais, como estrutura de propriedade, governança corporativa e gerenciamento de resultados.

Badolato, P. G., Donelson, D. C., \& Ege, M. (2014). Audit committee financial expertise and earnings management: The role of status. Journal of Accounting and Economics, 58(2-3), 208-230.

Barney, J. (1991). Firm resources and sustained competitive advantage. Journal of management, 17(1), 99-120.

Barney, J. B. (1996). The resource-based theory of the firm. Organization science, 7(5), 469-469.

Bartoloni, E. (2013). Capital structure and innovation: causality and determinants. Empirica, 4O(1), 111-151.

Bastos, L. T., Feldmann, P. R., \& Fouto, N. M. M. D. (2014). Inovação de varejo sustentável: e-commerce e comércio justo. Revista de Administração da Universidade Federal de Santa Maria, 7, 88-99.

Benetti, K., Benetti, K., Utzig, M. J. S., Braun, M., \& Oro, I. M. (2014). Evidenciação de subvenção e assistência governamentais das empresas na BM\&FBOVESPA. Revista Evidenciação Contábil \& Finanças, 2(1), 75-90.

Blazsek, S., \& Escribano, A. (2016). Patent propensity, R\&D and market competition: Dynamic spillovers of innovation leaders and followers. Journal of Econometrics, 191(1), 145-163. 
Block, J. H., Kohn, K., Miller, D., \& Ullrich, K. (2015). Necessity entrepreneurship and competitive strategy. Small Business Economics, 44(1), 37-54.

Boehe, D. M., Larentis, F., De Toni, D., \& Mattia, A. Á. (2011). Papel das relações interorganizacionais e da capacidade de inovação na propensão para exportar. REAd-Revista Eletrônica de Administração, 17(1), 86116.

Boermans, M. A., \& Roelfsema, H. (2012). A resource-based view of internationalization in emerging economies. In Impacts of Emerging Economies and Firms on International Business (pp. 13-31). Palgrave Macmillan, London.

Borini, F. M., Floriani, D. E., \& Fleury, M. T. L. (2012). The relation between size and development of organizational competencies in Brazilian multinationals. Revista de Administração (São Paulo), 47(4), 596-608.

Botelho, D., \& Guissoni, L. (2016). Varejo: competitividade e inovação. Revista de Administração de Empresas, 56(6), 596-599.

Brito, L. A. L., Brito, E. P. Z., \& de Vasconcelos, F. C. (2007). Ser uma empresa grande é vantagem para crescer?. Revista de Administração FACES Journal, 6(2), 61-75.

Brito, L. A. L., \& Vasconcelos, F. C. D. (2009). The variance composition of firm growth rates. BARBrazilian Administration Review, 6(2), 118-136.

Brito, R. P., \& Brito, L. A. L. (2012). Vantagem competitiva, criação de valor e seus efeitos sobre o desempenho. RAE-Revista de Administração de Empresas, 52(1), 70-84.

Brock, D. M., \& Yaffe, T. (2008). International diversification and performance: The mediating role of implementation. International Business Review, 17(5), 600-615.

Brown, N. C., \& Kimbrough, M. D. (2011). Intangible investment and the importance of firm-specific factors in the determination of earnings. Review of Accounting Studies, 16(3), 539-573.

Bushman, R. M., \& Piotroski, J. D. (2006). Financial reporting incentives for conservative accounting: The influence of legal and political institutions. Journal of Accounting and Economics, 42(1-2), 107-148.
Carmo, C. H. S., Ribeiro, A. M., \& De Carvalho, L. N. G. (2011). Convergência de fato ou de direito? A influência do sistema jurídico na aceitação das normas internacionais para pequenas e médias empresas. Revista Contabilidade \& Finanças, 22(57), 242-262.

Carneiro, J. M. T., \& Dib, L. A. (2007). Avaliação comparativa do escopo descritivo e explanatório dos principais modelos de internacionalização de empresas. Revista Eletrônica de Negócios Internacionais, 2(1), 125.

Castro, B. H. R. (2011). Influência da estruturação de departamentos de P\&D na inovação: um estudo na indústria de máquinas e implementos agrícolas no Brasil. RAl-Revista de Administração e Inovação, 8(1), 196-220.

Cheng, C. C., Yang, C. L., \& Sheu, C. (2014). The link between eco-innovation and business performance: a Taiwanese industry context. Journal of Cleaner Production, 64, 81-90.

Chi, C. W., Hung, K., Cheng, H. W., \& Lieu, P. T. (2015). Family firms and earnings management in Taiwan: Influence of corporate governance. International Review of Economics \& Finance, 36, 88-98.

Cho, H. J., \& Pucik, V. (2005). Relationship between innovativeness, quality, growth, profitability, and market value. Strategic management journal, 26(6), 555-575.

Conto, S. M., Antunes Júnior, J. A. V., \& Vaccaro, G. L. R. (2016). A inovação como fator de vantagem competitiva: estudo de uma cooperativa produtora de suco e vinho orgânicos. Gestão \& Produção, 23(2), 397407.

Coronel, D. A., Azevedo, A. F. Z., \& Campos, A. C. (2014). Política industrial e desenvolvimento econômico: a reatualização de um debate histórico. Revista de Economia Política, 34(1), 103-119.

Costa, C., Lages, L. F., \& Hortinha, P. (2015). The bright and dark side of CSR in export markets: Its impact on innovation and performance. International Business Review, 24(5), 749-757.

Dai, L., Maksimov, V., Gilbert, B. A., \& Fernhaber, S. A. (2014). Entrepreneurial orientation and international scope: The differential roles of innovativeness, proactiveness, and risk-taking. Journal of Business Venturing, 29(4), 511-524. 
De Paula, R. M., Ferreira, M. P., \& Quinte, P. S. (2019). Aplicação das teorias de internacionalização em pequenas empresas. Revista PRETEXTO, 20(2), 32-47.

Dhaliwal, D. S., Gleason, C. A., Heitzman, S., \& Melendrez, K. D. (2008). Auditor fees and cost of debt. Journal of accounting, auditing \& finance, 23(1), 1-22.

Fávero, L. P., \& Belfiore, P. (2017). Métodos quantitativos com Stata: procedimentos, rotinas e análise de resultados. São Paulo: Elsevier Brasil.

Fernandes, C. M. A., Bandeira-de-Mello, R., \& Zanni, P. P. (2012). The role of political factors in the internationalization of companies: the case of Energias de Portugal (EDP) in Brazil. Cadernos EBAPE. BR, 10(2), 435-455.

Floriani, D. E., \& Fleury, M. T. (2012). O efeito do grau de internacionalização nas competências internacionais e no desempenho financeiro da PME brasileira. Revista de Administração Contemporânea, 16(3), 438-458.

Geiger, S. W., \& Makri, M. (2006). Exploration and exploitation innovation processes: The role of organizational slack in R \& D intensive firms. The Journal of High Technology Management Research, 17(1), 97108.

Gelfand, M. J., Erez, M., \& Aycan, Z. (2007). Crosscultural organizational behavior. Annu. Rev. Psychol., 58, 479-514.

Giroud, X., \& Mueller, H. M. (2010). Does corporate governance matter in competitive industries?. Journal of Financial Economics, 95(3), 312-331.

Gomes, C. M., Kruglianskas, I., \& Scherer, F. L. (2011). Innovation management for sustainable development practices in the internalization context. Journal of technology management \& innovation, 6(2), 111-127.

Gonçalves Filho, C., Veit, M. R., \& Monteiro, P. R. R. (2013). Inovação, estratégia, orientação para o mercado e empreendedorismo: identificação de clusters de empresas e teste de modelo de predição do desempenho nos negócios. RAl-Revista de Administração e Inovação, 10(2), 81-101.

Guillen, M. F. (2000). Business groups in emerging economies: A resource-based view. Academy of Management Journal, 43(3), 362-380.

Halilem, N., Amara, N., \& Landry, R. (2014). Exploring the relationships between innovation and internationalization of small and medium-sized enterprises: A nonrecursive structural equation model.
Canadian Journal of Administrative Sciences/Revue Canadienne des Sciences de l'Administration, 31(1), 1834.

Hassel, A., Höpner, M., Kurdelbusch, A., Rehder, B., \& Zugehör, R. (2003). Two dimensions of the internationalization of firms. Journal of Management Studies, 40(3), 705-723.

House, R. J., Hanges, P. J., Javidan, M., Dorfman, P. W., \& Gupta, V. (Eds.). (2004). Culture, leadership, and organizations: The GLOBE study of 62 societies. Sage publications.

Huguet, D., \& Gandía, J. L. (2016). Audit and earnings management in Spanish SMEs. BRQ Business Research Quarterly, 19(3), 171-187.

Hurley, R. F., \& Hult, G. T. M. (1998). Innovation, market orientation, and organizational learning: an integration and empirical examination. The Journal of marketing, 62, 42-54.

Javalgi, R. R. G., Griffith, D. A., \& Steven White, D. (2003). An empirical examination of factors influencing the internationalization of service firms. Journal of Services Marketing, 17(2), 185-201.

Jayaram, J., Oke, A., \& Prajogo, D. (2014). The antecedents and consequences of product and process innovation strategy implementation in Australian manufacturing firms. International Journal of Production Research, 52(15), 4424-4439.

Jung, J. C., \& Bansal, P. (2009). How firm performance affects internationalization. Management International Review, 49(6), 709-732.

Kayo, E. K., Teh, C. C., \& Basso, L. F. C. (2006). Ativos intangíveis e estrutura de capital: a influência das marcas e patentes sobre o endividamento. Revista de Administração-RAUSP, 41(2).

Khanna, T., \& Rivkin, J. W. (2001). Estimating the performance effects of business groups in emerging markets. Strategic management journal, 22(1), 45-74.

Knight, G. A., \& Cavusgil, S. T. (2004). Innovation, organizational capabilities, and the born-global firm. Journal of international business studies, 35(2), 124-141.

Knight, G. A., \& Liesch, P. W. (2016). Internationalization: From incremental to born global. Journal of World Business, 51(1), 93-102.

Kull, T. J., \& Wacker, J. G. (2010). Quality management effectiveness in Asia: The influence of culture. Journal of operations management, 28(3), 223239. 
La Porta, R., Lopez-de-Silanes, F., Shleifer, A., \& Vishny, R. W. (1998). Law and finance. Journal of political economy, 106(6), 1113-1155.

Lima, A. C., \& Carmona, C. U. (2010). Determinantes da formação do capital intelectual nas empresas produtoras de tecnologia da informação e comunicação. Revista de Administração Mackenzie (Mackenzie Management Review), 12(1).

Lopes, I. F., Beuren, I. M., \& Dametto, I. D. R. B. (2016). Evidenciação dos recursos aplicados em pesquisa, desenvolvimento \& inovação e da redução de carga tributária por empresas listadas na BM\&FBovespa. ConTexto, 16(32).

Machado, D. D. P. N., \& Carvalho, L. C. (2013). Ambiente favorável ao desenvolvimento de inovações: proposição de um modelo de análise organizacional. Revista de Administração-RAUSP, 48(3).

Maia, A. B. G. R., de Vasconcelos, A. C., \& De Luca, M. M. M. (2013). Governança corporativa e internacionalização do capital social das companhias brasileiras do setor de construção e transportes. Internext, 8(2), 40-60.

Mazzioni, S., Rigo, V. P., Klann, R. C., \& Silva Junior, J. C. A. (2014). A relação entre a intangibilidade e o desempenho econômico: Estudo com empresas de capital aberto do Brasil, Rússia, Índia, China e África do Sul (BRICS). Advances in Scientific and Applied Accounting, 7(1), 122-148.

Miranda, K. F., Vasconcelos, A. C., De Luca, M. M. M., \& Cabral, J. E. O. (2015). A capacidade inovativa e o desempenho econômico-financeiro de empresas inovadoras brasileiras. REAd-Revista Eletrônica de Administração, 21(2).

Mitter, C., Duller, C., Feldbauer-Durstmüller, B., \& Kraus, S. (2014). Internationalization of family firms: the effect of ownership and governance. Review of Managerial Science, 8(1), 1-28.

Moura, G. D., Mecking, D. V., \& Scarpin, J. E. (2013). Market competitiveness, intangible assets and efficiency in combination of fixed assets in companies listed on the BM \& F Bovespa. Enfoque, 32(3), 19.

Moura, P. G., \& Galina, S. V. (2009). Empresas multinacionais de origem brasileira e a publicação internacional de patentes. RAl-Revista de Administração e Inovação, 6(3), 26-45.

Muzychenko, O., \& Liesch, P. W. (2015). International opportunity identification in the internationalisation of the firm. Journal of World Business, 50(4), 704-717.
Nekhili, M., Boubaker, S., \& Lakhal, F. (2012). Ownership structure, voluntary R\&D disclosure and market value of firms: the French case. International Journal of Business, 17(2), 126.

Naor, M., Jones, J. S., Bernardes, E. S., Goldstein, S. M., \& Schroeder, R. (2014). The culture-effectiveness link in a manufacturing context: A resource-based perspective. Journal of World Business, 49(3), 321-331.

O'brien, J. P. (2003). The capital structure implications of pursuing a strategy of innovation. Strategic Management Journal, 24(5), 415-431.

OCDE - Organização para a Cooperação e Desenvolvimento Econômico. (2018). Manual de Frascati 2007: proposta de práticas exemplares para inquéritos sobre investigação e desenvolvimento experimental. OCDE. Disponível em: http://www.uesc.br/nucleos/nit/manualfrascati.pdf.

OCDE - Organização para a Cooperação e Desenvolvimento Econômico (2005). Manual de Oslo: diretrizes para coleta e interpretação de dados sobre inovação. OCDE.

Oliveira, T., Thomas, M., \& Espadanal, M. (2014). Assessing the determinants of cloud computing adoption: An analysis of the manufacturing and services sectors. Information \& Management, 51(5), 497-510.

Pangarkar, N. (2008). Internationalization and performance of small-and medium-sized enterprises. Journal of world business, 43(4), 475-485.

Perez, M. M., \& Famá, R. (2015). Características estratégicas dos ativos intangíveis e o desempenho econômico da empresa. Unisanta Law and Social Science, 4(2), 107-123.

Platchek, R. B., Floriani, D. E., \& Borini, F. M. (2012). A influência do grau de internacionalização no desempenho das empresas têxteis. Revista Gestão Organizacional, 5(1), 70-81.

Porter, M. E. (1996). Competitive advantage, agglomeration economies, and regional policy. International regional science review, 19(1-2), 85-90.

Prajogo, D. I. (2016). The strategic fit between innovation strategies and business environment in delivering business performance. International Journal of Production Economics, 171, 241-249.

Reynoso, C. F., \& Figueroa, L. E. O. (2010). Intangible resources as a determinant of accelerated internationalization. Global Journal of Business Research 4(4), 95-105. 
Ribeiro, P. D. (2016). Relação entre internacionalização e inovação nas empresas: um estudo de caso. Revista do BNDES, 46(1), 263-309.

Rodrigues, L. C., Rechziegel, W., Bastos, N. G., Fiorillo, A., \& Santos, J. N. (2012). Inovação aberta e internacionalização de negócio. Revista Pretexto, 13(3).

Ruigrok, W., \& Wagner, H. (2003). Internationalization and performance: An organizational learning perspective. MIR: Management International Review, 63-83.

Santos, J. G. C., Vasconcelos, A. C., \& De Luca, M. M. M. (2015). Internacionalização de empresas e governança corporativa: uma análise das maiores companhias abertas do Brasil. Advances in Scientific and Applied Accounting, 8(3), 300-319.

Santos, J. G. C., Vasconcelos, A. C., \& De Luca, M. M. M. (2013). Perfil da inovação e da internacionalização de empresas transnacionais. RAl-Revista de Administração e Inovação, 10(1), 189-211.

Schreiber, D., Bessi, V. G., Puffal, D. P., \& Tondolo, V. A. G. (2013). Posicionamento estratégico de MPE'S com base na inovação através do modelo hélice tríplice. Revista Eletrônica de Administração, 76(3), 767-795.

Schumpeter, J. A. (1988). Capitalismo, sociedade e democracia. São Paulo: Abril Cultural.

Sharma, V. M., \& Erramilli, M. K. (2004). Resourcebased explanation of entry mode choice. Journal of Marketing theory and Practice, 12(1), 1-18.

Shearmur, R., Doloreux, D., \& Laperrière, A. (2015). Is the degree of internationalization associated with the use of knowledge intensive services or with innovation?. International business review, 24(3), 457-465.

Shefer, D., \& Frenkel, A. (2005). R\&D, firm size and innovation: an empirical analysis. Technovation, 25(1), 25-32.

Stal, E. (2010). Internacionalização de empresas brasileiras e o papel da inovação na construção de vantagens competitivas. RAl-Revista de Administração e Inovação, 7(3), 120-149.
Tavassoli, S., \& Karlsson, C. (2015). Persistence of various types of innovation analyzed and explained. Research Policy, 44(10), 1887-1901.

Teece, D. J., Pisano, G., \& Shuen, A. (1997). Dynamic capabilities and strategic management. Strategic Management Journal, 18(7), 509-533.

Teh, C. C., Kayo, E. K., \& Kimura, H. (2008). Marcas, patentes e criação de valor. Revista de Administração Mackenzie (Mackenzie Management Review), 9(1), 86106.

Terra, N. M., Barbosa, J. G. P., \& Bouzada, M. A. C. (2015). A influência da inovação em produtos e processos no desempenho de empresas brasileiras. RA/Revista de Administração e Inovação, 12(3), 183-208.

Terziovski, M. (2010). Innovation practice and its performance implications in small and medium enterprises (SMEs) in the manufacturing sector: a resource-based view. Strategic Management Journal, 31(8), 892-902.

Trąpczyński, P., \& Wrona, T. (2013). From going international to being international-strategies for international competitiveness. Poznan University of Economics Review, 13(1), 89-114.

Vargas, M. I. R. (2015). Determinant factors for small business to achieve innovation, high performance and competitiveness: organizational learning and leadership style. Procedia-Social and Behavioral Sciences, 169, 4352.

Wang, L. (2014). Internationalization with Chinese characteristics: The changing discourse of internationalization in China. Chinese Education \& Society, 47(1), 7-26.

Wernerfelt, B. (1984). A resource-based view of the firm. Strategic management journal, 5(2), 171-180.

Zemplinerová, A., \& Hromádková, E. (2012). Determinants of firm's innovation. Prague Economic Papers, 21(4), 487-503.

Zen, A. C. (2012). O processo de internacionalização e o impacto nos recursos da firma: o caso da vinícola Casa Valduga. Internext, 7(1), 123-148. 
Sobre os Autores:

Antonio Rodrigues Albuquerque Filho - Universidade Federal do Ceará - UFCE, Fortaleza, Ceará, (Brasil). E-mail: antoniofilhoufc@hotmail.com Orcid id: https://orcid.org/0000-0003-2108-3979

Maria Macileya Azevedo Freire - Universidade Federal do Ceará - UFCE, Fortaleza, Ceará, (Brasil). E-mail: macileya@hotmail.com Orcid id: https://orcid.org/0000-0002-8167-0473

Marcia Martins Mendes De Luca - Universidade Federal do Ceará - UFCE, Fortaleza, Ceará, (Brasil). E-mail: marciammdeluca@gmail.com Orcid id: https://orcid.org/0000-0002-9995-5637

Alessandra Carvalho de Vasconcelos - Universidade Federal do Ceará - UFCE, Fortaleza, Ceará, (Brasil). E-mail: alevasconcelos.ufc@gmail.com Orcid id: https://orcid.org/0000-0002-6480-5620

\title{
INFLUENCE OF INTERNATIONALIZATION AND INNOVATION ON CORPORATE COMPETITIVENESS
}

\author{
Antonio Rodrigues Albuquerque Filho, Maria Macileya Azevedo Freire, Marcia Martins Mendes De Luca, \\ Alessandra Carvalho de Vasconcelos \\ Universidade Federal do Ceará - UFCE, Fortaleza, Ceará, (Brasil)
}

\section{ARTICLE DETAILS}

\section{Article history:}

Received: 31 January 2019

Accepted: 08 September 2019

Available online January: 01 th 2020

Double Blind Review System

Scientific Editor

Ilan Avrichir

\section{Key words}

Internationalization

Innovation

Competitiveness

BRICS

\begin{abstract}
RESUMO
Objective: In light of Resource-Based View, we evaluated the influence of internationalization and innovation on corporate competitiveness in firms from BRICS countries traded on the NYSE.

Methods: In this descriptive and quantitative study, panel data from 64 nonfinancial firms from BRICS countries traded on the NYSE in the period 2015-2017 were submitted to testing for differences between means, Pearson correlation analysis and multiple linear regression.

Main results: The results of the regressions were consistent with the findings from the testing for differences between means and the Pearson correlation analysis, having failed to detect a significant association between the study variables (internationalization and innovation) and corporate competitiveness. However, the presence of innovation strategies had a relevant impact on profitability, indicating that the greater the profitability, the smaller the competitiveness.

Relevance/originality: Internationalization and innovation are frequent topics in the literature, but the combination of these two constructs and their influence on corporate competitiveness-arguably a topic of considerable economic relevance to organizations-has not been sufficiently explored. Moreover, few empirical studies have evaluated competitiveness proxied by the Herfindahl-Hirschman index.

Theoretical/methodological contributions: Our study makes a significant methodological contribution by employing internationalization and innovation as multidimensional metrics in the analysis of the ability of international involvement and strategic resource adaptation to generate competitive advantage.
\end{abstract}




\title{
INFLUENCIA DE LA INTERNACIONALIZACIÓN Y DE LA INNOVACIÓN EN LA COMPETITIVIDAD EMPRESARIAL
}

\author{
Antonio Rodrigues Albuquerque Filho, Maria Macileya Azevedo Freire, Marcia Martins Mendes De Luca, \\ Alessandra Carvalho de Vasconcelos \\ Universidade Federal do Ceará - UFCE, Fortaleza, Ceará, (Brasil)
}

DETALLES DEL ARTÍ́CULO

Historia del Artículo:

Recibido: 31 Enero 2019

Aceptado: 08 Septiembre 2019

Disponible en línea: 01 de enero 2020

Double Blind Review System

\section{Editor Científico}

Ilan Avrichir

Palabras-clave:

Internacionalización

Innovación

Competitividad

BRICS

\begin{abstract}
RESUMO
Objetivo: Investigar la influencia de la internacionalización y de la innovación en la competitividad en empresas de los países BRICS que figuran en Nyse, desde la perspectiva de VBR.

Método: De naturaleza descriptiva y enfoque cuantitativo, la investigación utiliza el test de diferencias entre medias, correlación y regresión lineal múltiple con datos en panel, para el análisis de datos de 2015 a 2017, de 64 empresas no financieras con sede en países BRICS enumerados en Nyse

Principales resultados: Corroborando los hallazgos constatados en los testes de diferencias entre medias y correlación de Pearson, los resultados de la regresión indican que los indicadores de internacionalización y de innovación no ejercen influencia sobre la competitividad de las empresas. Sin embargo, se resalta que, en la presencia de estrategias de innovación, la rentabilidad se muestra relevante, denotando que, a mayor rentabilidad de la organización, menor será su poder de competitividad.

Relevancia/originalidad: A pesar de que individualmente la internacionalización y la innovación son temas ampliamente discutidos en la literatura, es relevante que sean explorados en la perspectiva de la competitividad empresarial, puesto que son esenciales para el fortalecimiento de la economía y señalados como de gran valor organizacional. Igualmente, pocos estudios analizaron empíricamente la competitividad teniendo como proxy el índice de Herfindahl-Hirschman.

Contribuciones teóricas/metodológicas: Se destaca la contribución del estudio al campo académico al ponderar la internacionalización y la innovación como métricas multidimensionales para análisis de la participación internacional de las empresas y de la adaptación estratégica de recursos como fuentes de ventaja competitiva.
\end{abstract}

\section{Para citar este artigo:}

Albuquerque Filho, A., Freire, M., De Luca, M., \& Vasconcelos, A. (2020). Influência da Internacionalização e da Inovação na Competitividade Empresarial. Internext, 15(1), 01-18. doi: http://dx.doi.org/10.18568/internext.v15i1.521 